\title{
REVIEW: A COHERENT AND COMPREHENSIVE MODEL OF THE EVOLUTION OF THE OUTER SOLAR SYSTEM
}

\author{
Alessandro Morbidelli \\ Departement Cassiopée: Université de Nice - Sophia Antipolis, Observatoire de la Côte d'Azur, CNRS. \\ 06304 Nice Cedex 4, France
}

Received by CRAS, on Sept 20, 2010; accepted by CRAS, on Oct 27, 2010 


\begin{abstract}
Since the discovery of the first extra-solar planets, we are confronted with the puzzling diversity of planetary systems. Processes like planet radial migration in gasdisks and planetary orbital instabilities, often invoked to explain the exotic orbits of the extra-solar planets, at first sight do not seem to have played a role in our system. In reality, though, there are several aspects in the structure of our Solar System that cannot be explained in the classic scenario of in-situ formation and smooth evolution of the giant planets. This paper describes a new view of the evolution of the outer Solar System that emerges from the so-called 'Nice model' and its recent extensions. The story provided by this model describes a very "dynamical" Solar System, with giant planets affected by both radial migrations and a temporary orbital instability. Thus, the diversity between our system and those found so far around other stars does not seem to be due to different processes that operated here and elsewhere, but rather stems from the strong sensitivity of chaotic evolutions to small differences in the initial and environmental conditions.
\end{abstract}

In press in "C.R. Physique de l'Académie des Sciences”.

\title{
1. Introduction
}

When looking at the structure of the outer Solar System, i.e. the four giant planets and the populations of small bodies from the orbit of Jupiter outwards, one sees several puzzling aspects that do not fit the simple scenario of in-situ formation of planets from a circum-solar disk of gas 
and solids, developed over the last centuries from the ideas of Laplace (Laplace, 1796). Moreover, our Solar System looks quite different from the planetary systems discovered so far around other stars.

For instance: (i) many extra-solar giant planets have small orbital radii, comparable (or smaller) than those of the terrestrial planets of our Solar System (Mercury to Mars); instead, our giant planets (Jupiter to Neptune) orbit the Sun at a distance of 5-30 times that of the Earth. (ii) Giant planets are expected to form on circular and co-planar orbits; however, the orbital eccentricities and inclinations of our giant planets, although small, are definitely much larger than expected from formation models; the orbits of the majority of the extra-solar giant planets are even more at odds with the theoretical expectations, because they are much more eccentric than the orbits of the planets of our system. (iii) Many extra-solar systems have planets in mutual mean motion resonances, where the ratio of the orbital periods is equal to a ratio of small integer numbers (often 1/2); but the orbits of the planets of our system do not have this property. (iv) One would expect to find, beyond the orbit of the last planet, a disk of small icy objects, called planetesimals, that preserves its original, virgin structure: quasi-circular and coplanar orbits and a cumulatively large total mass; instead the Kuiper belt (the population of icy bodies tha have been found beyond the orbit of Neptune) is in total less massive than our Moon, it has an abrupt outer edge at the location of the $1 / 2$ resonance with Neptune and the eccentricities and inclinations of its objects can be as large as allowed by stability constraints. (v) One would expect that the Solar System evolved gradually, from a primordial chaos characterized by mutual collisions and ejections of bodies, to the current state of essentially regular orbital motion; however, the terrestrial planets, the asteroids and, possibly, the satellites of the giant planets, carry the scars of a "Late Heavy Bombardment" (LHB), suddenly triggered 600 million years after planet formation, 
or approximately 3.9 Gy (Giga-year) ago. This argues for a sudden change in the structure of the Solar System, so that a stable reservoir of small bodies became unstable and its objects started to intersect those of the planets and collide with the latter.

The 'Nice model' -so named because it was developed at the Observatoire de la Côte d'Azur in Nice- has the ambition to explain all these and other intriguing features in the framework of a unitary scenario. Several other models have been developed over the years to explain one or another of the puzzling properties of our Solar System, but none has the comprehensive character of the Nice model.

In this paper, I will review the basic ideas behind this model. In section 2 , I will present the original version of the model, as proposed in 2005. The model has vastly evolved since then, in order to overcome its limitations and extend the time-span of the events that it can describe. I will discuss these evolutions in Section 3. Section 4 will summarize the current view of Solar System evolution that emerges from this model.

\section{The original model}

The original Nice model was developed to explain the origin of the small, but non-negligible eccentricities and inclinations of the giant planets and the origin of the Late Heavy Bombardment of the inner Solar System.

Like most models, the Nice model was based on pre-existing ideas. First, it was known since Fernandez and Ip (1984) that, after the disappearance of the gas, while scattering away the primordial planetesimals from their neighboring regions, the giant planets had to migrate in 
semi-major axis as a consequence of angular momentum conservation. Given the configuration of the giant planets in our Solar System, this migration should have had a general trend. Uranus and Neptune have difficulty ejecting planetesimals onto hyperbolic orbits. Apart from the few percent of planetesimals that they can permanently store in the Oort cloud (the shell-like reservoir of long-period comets, situated at about $10^{4}$ Astronomical Units (AU) from the Sun; Dones et al., 2004), or emplace onto long-lived orbits in the trans-Neptunian region (Duncan and Levison, 1997), the large majority of the planetesimals that are under the influence of Uranus and Neptune are eventually scattered inwards, towards Saturn and Jupiter. Thus, Uranus and Neptune, by reaction, have to move outwards. Jupiter, on the other hand, eventually ejects from the Solar System almost all of the planetesimals that it encounters: thus it has to move inwards. The fate of Saturn is more difficult to predict, a priori. However, modern numerical simulations show that this planet also moves outwards, although only by a few tenths of an AU for reasonable disk's masses (e.g. 50 Earth masses; see Hahn and Malhotra, 1999; Gomes et al., 04).

Second, it was known that planets embedded in a planetesimal disk suffer "dynamical friction" which damps their orbital eccentricities and inclinations (Wetherill and Stewart, 1993). Thus, the planetesimal scattering process that leads to planet migration by itself cannot enhance the eccentricities and inclinations of the planets relative to their (almost null) initial values (Morbidelli et al., 2009). However, it was also known that, if the planets cross mutual mean motion resonances during their divergent migration, their eccentricities are enhanced almost impulsively (Chiang, 2003). The eccentricity increase depends on the planetary masses and on the resonance involved. If the eccentricities become too large, then planets can become unstable.

Third, it was shown by Thommes et al. (1999) that the instability of the giant planets system would not necessarily lead to the disruption of the outer Solar System. In several cases, 
Uranus and Neptune are scattered outwards by Jupiter and Saturn; then the interaction with the disk of planetesimals can damp by dynamical friction the eccentricities of Uranus and Neptune, preventing them to have further close encounters with Jupiter or Saturn and between themselves; consequently, the 4-planet system can achieve a new stable configuration.

Last, Levison et al. (2001) showed that the dispersal of a planetesimal disk of $\sim 50$ Earth masses by the migrating giant planets would induce a bombardment of the terrestrial planets of magnitude comparable to that of the LHB; thus the problem of the origin of the LHB is re-conduced to the problem of finding a plausible mechanism for triggering giant planet migration at a correspondingly late time. 1$]$

Building on all these results, the Nice model postulated that, at the time of the dissipation of the gas-disk, the four fully-grown giant planets were in a compact configuration, with quasi-circular, coplanar orbits (as predicted by planet formation models) and with orbital radii ranging from 5.5 to $17 \mathrm{AU}$; Saturn and Jupiter were close enough to each other to have a ratio of orbital periods smaller than 2 (Tsiganis et al., 2005; the current ratio of their orbital periods is almost 2.5). During their planetesimal-driven divergent migration, Saturn and Jupiter increased their orbital period ratio. Thus, with the adopted initial conditions, Saturn and Jupiter eventually crossed their mutual 1/2 mean-motion resonance (which occurs when the period ratio is exactly 2). This resonance enhances the eccentricities of Jupiter and Saturn, enough to make the whole

\footnotetext{
${ }^{1}$ It was proposed in Levison et al. (2001) that this mechanism was the late formation of Uranus and Neptune, but a formation as late as $600 \mathrm{My}$ is inconsistent with the physical structure of these planets (which contain hydrogen and helium in roughly solar proportion) and with their dynamics during accretion (Levison et al., 2007).
} 
4-planet system unstable. The dynamics then evolves through mutual scattering among the planets and dynamical friction exerted by the disk, as described above. Eventually a new stable configuration is achieved once all disk particles are dispersed and removed. The simulations in Tsiganis et al. (2005) show that, if the planetesimal disk contained about 35 Earth masses and was truncated at $\sim 35 \mathrm{AU}$, this dynamical evolution leads to a final orbital configuration of the planetary system that reproduces the current configuration remarkably well, in terms of semi-major axes, eccentricities and inclinations (see Fig. 1).

With this result in hands, Gomes et al. (2005) could put all the elements together in a coherent scenario for the LHB origin. They reasoned that, at the end of the gas-disk phase, the planetesimal disk should have contained only those bodies that had dynamical lifetimes longer than the lifetime of the solar nebula (a few million years), because the planetesimals initially on orbits with shorter dynamical lifetimes should have been eliminated earlier, during the nebula era. Assuming the initial planetary system of Tsiganis et al. (2005), this constraints the planetesimal disk to start about 1 AU beyond the position of the last planet. With this kind of disk, the 1/2 resonance crossing event that destabilizes the planetary system occurs in the simulations of Gomes et al. (2005) at a time ranging from $192 \mathrm{My}$ to $875 \mathrm{My}$. Modifying the initial planetary orbits also leads to changes in the resonance crossing time, pushing it up to $1.1 \mathrm{~Gy}$ after the beginning of the simulation. This range of instability times brackets well the date of the LHB, as estimated from lunar data.

The top panel of Fig. 2 shows the giant planets' evolution in a representative simulation of Gomes et al. (2005). Initially, the giant planets migrated slowly due to the leakage of particles from the disk. This phase lasted $875 \mathrm{My}$, at which point Jupiter and Saturn crossed their 1/2 resonance. At the resonance crossing event, as in Tsiganis et al. (2005), the orbits of the ice 


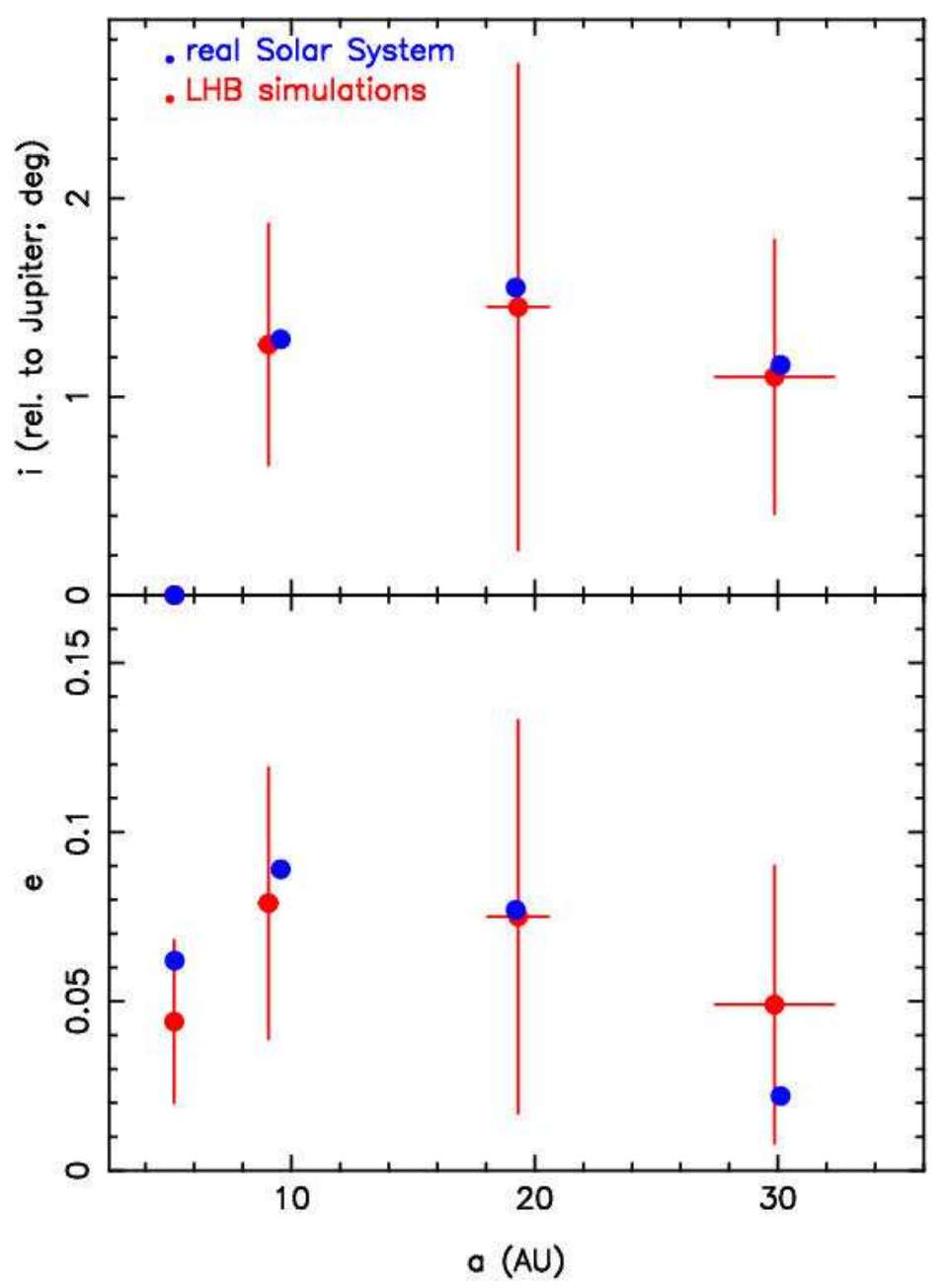

Fig. 1.- Comparison of the synthetic final planetary systems obtained in Tsiganis et al. (2005) with the real outer Solar System. Top: Proper eccentricity vs. semi-major axis. Bottom: Proper inclination vs. semi-major axis. Here, proper eccentricities and inclinations are defined as the maximum values acquired over a 2 My time-span and were computed from numerical integrations. The inclinations are measured relative to Jupiters orbital plane. The values for the real planets are presented as filled blue dots. The red dots mark the mean of the proper values for 15 simulations. The error bars represent one standard deviation of the measurements. 


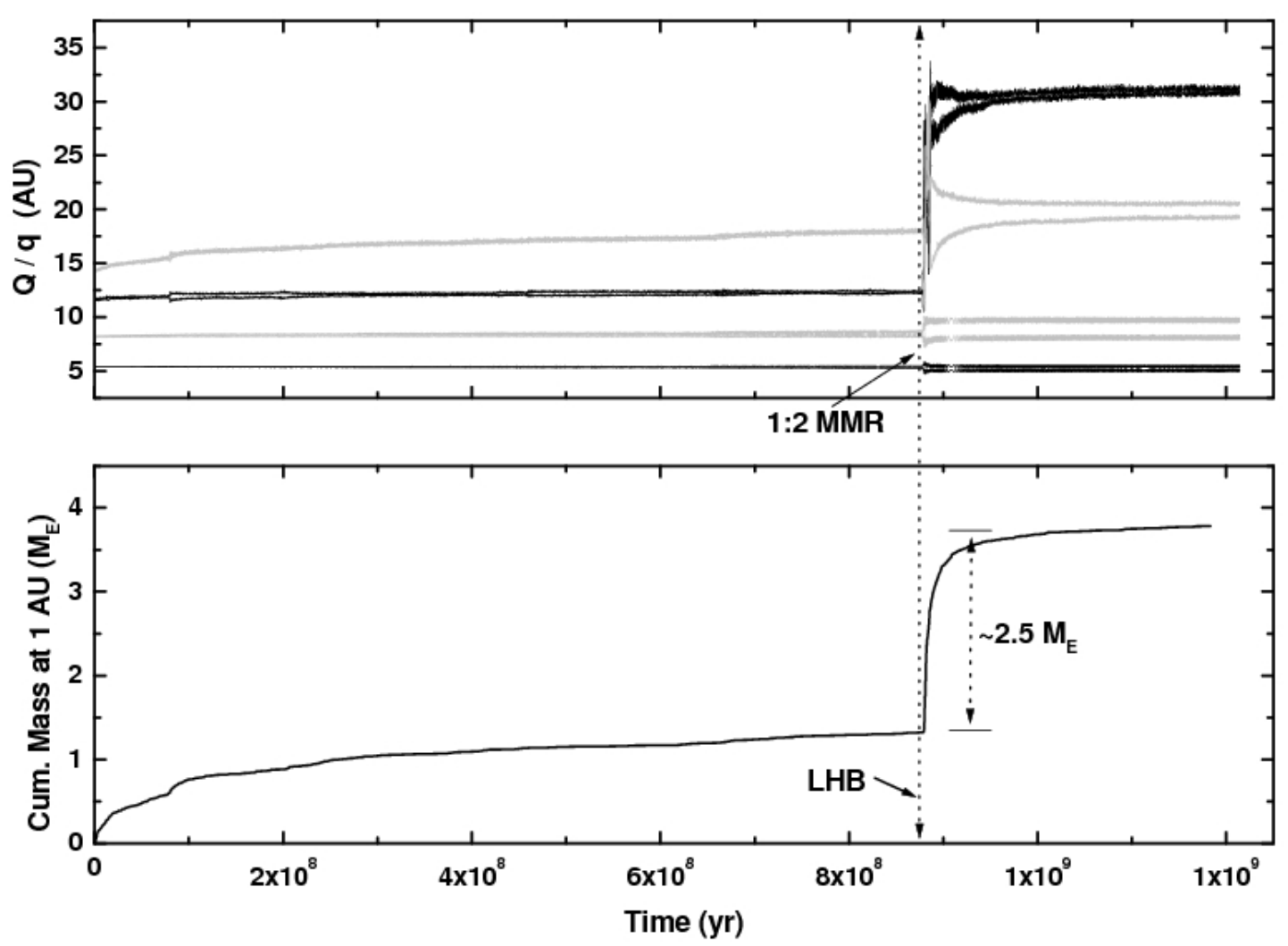

Fig. 2.- Planetary migration and the corresponding mass flux towards the inner Solar System, from a representative simulation of Gomes et al. (2005). Top: the evolution of the 4 giant planets. Each planet is represented by a pair of curves - the aphelion and perihelion distances. In this simulation Jupiter and Saturn cross their 1/2 mean-motion resonance at $880 \mathrm{My}$. Bottom: the cumulative mass of comets (solid curve) and asteroids (dashed curve) accreted by the Moon. The comet curve is offset so that the value is zero at the time of $1 / 2$ resonance crossing. 
giants became unstable and they were scattered into the disk by Saturn. They disrupted the disk and scattered objects all over the Solar System, including the inner regions. Eventually they stabilized on orbits very similar to the current ones, at $\sim 20$ and $\sim 30$ AU respectively. The solid curve in the bottom panel shows the amount of material from the primordial trans-Neptunian disk that struck the Moon, as a function of time. The amount of material hitting the Moon after the resonance crossing event is consistent with the mass $\left(6 \times 10^{21} \mathrm{~g}\right)$ estimated from the number and size distribution of lunar basins that formed around the LHB epoch (Levison et al., 2001).

However, the planetesimals from the distant disk -which can be identified as 'comets'were not the only ones to hit the terrestrial planets. The radial migration of Jupiter and Saturn forced secular resonances (resonances between the precession periods of the asteroids and of the giant planets) to sweep across the asteroid belt, exciting the eccentricities and the inclinations of asteroids. The fraction of the main belt population that acquired planet-crossing eccentricities depends quite crucially on the orbital distribution that the belt had before the LHB, which is not well known. According to the simulations in O'brien et al. (2007), at the end of the terrestrial planet formation process, which pre-dates the LHB, the asteroid belt should have had a dynamical excitation comparable, or slightly larger than the current one. In these conditions of orbital excitation, the secular resonance sweeping at the time of the LHB would have left $\sim 5-10 \%$ of the objects in the asteroid belt (Gomes et al., 2005). Thus, at the LHB time, the asteroid belt would have been 10-20 times more massive than now. In this case, the total mass of the asteroids hitting the Moon would have been comparable to that of the comets (see Fig. 2). 


\subsection{Other successes of the Nice model}

To validate or reject a model, it is important to look at the largest possible number of constraints. Three populations immediately come to mind when considering the Nice model: the Trojans and the satellites of the giant planets and the Kuiper belt. Are their existence and structure consistent with the Nice scenario?

\section{TROJANS}

Jupiter and Neptune have a conspicuous populations of Trojan objects. These bodies, usually referred to as 'asteroids', follow essentially the same orbit as the planet, but lead or trail that planet by an angular distance of $\sim 60$ degrees, librating around the Lagrange triangular equilibrium points. The latter are the two positions where a small object, affected only by the gravity of the Sun and of one planet, can be stationary and stable relative to two larger objects; together with the positions of the Sun and the planet, they form two equilateral triangles, rotating in space (Lagrange, 1787).

To date, the number of known Jupiter Trojans is 4526. Probably all those larger than about $20 \mathrm{~km}$ in diameter are now known; they are about 1,000 objects. Instead, only seven Trojan of Neptune are now known, but detection statistics imply that the Neptune Trojan population could be comparable in number to that of Jupiter, and possibly even ten times larger (Chiang and Lithwick, 2005).

The simulations in Tsiganis et al. (2005) and Gomes et al. (2005) led to the capture of several particles on long-lived Neptunian Trojan orbits ( 2 per run, on average, with a lifetime larger than $80 \mathrm{My})$. Their eccentricities, during their evolution as Trojans, reached values smaller than 0.1 . 
These particles were eventually removed from the Trojan region, but this is probably an artifact of the graininess of Neptune's migration in the simulation, due to the quite large individual mass of the planetesimals.

Jovian Trojans are a more subtle issue that was addressed in detail in Morbidelli et al. (2005). There is a serious argument in the literature against the idea that Jupiter and Saturn crossed their 1/2 mean-motion resonance: if the crossing had happened, any pre-existing Jovian Trojans would have become violently unstable, and Jupiter's co-orbital region would have emptied (Gomes, 1998; Michtchenko et al., 2001). However, the dynamical evolution of a gravitating system of objects is time reversible. Thus, if the original objects can escape the Trojan region when it becomes unstable, other bodies can enter the same region and be temporarily trapped. Consequently, a transient Trojan population can be created if there is an external source of objects. In the framework of the Nice model, the source consists of the very bodies that are forcing the planets to migrate, which must be a large population given how far the planets must migrate. When Jupiter and Saturn move far enough from the 1/2 resonance that the co-orbital region becomes stable, the population that happens to be there at that time remains trapped. It then becomes the population of permanent Jovian Trojans still observable today.

This possibility has been tested with numerical simulations in Morbidelli et al. (2005). It was shown that the population of captured Trojans is consistent, in terms of total mass and orbital distribution, with the real population. In particular, the Nice model is the only model proposed so far which explains the inclination distribution of the Jovian Trojans. The origin of this distribution was considered to be the hardest problem in the framework of the classical scenario, according to which the Trojans formed locally and were captured at the time of Jupiter's growth (Marzari et al., 2002). 


\section{IRREGULAR SATELLITES}

The known irregular satellites of the giant planets are dormant comet-like objects that reside on stable prograde and retrograde orbits at large distances from the central object, where planetary perturbations are only slightly larger than solar ones.

One particularity of the irregular satellite systems is that, once the orbital radii are scaled relative to the radius of the sphere of gravitational influence of the respective planets, they are all very similar to each other (Jewitt and Sheppard, 2002). This invalidates the most popular models proposed for their origin, i.e. (i) capture due to the sudden growth of the giant planets (Heppenheimer and Porco, 1977) and (ii) capture due to gas drag in the primordial extended atmosphere of the giant planets (Cuk and Burns, 2004; Kortenkamp, 2005). In fact, Jupiter and Saturn are very different from Uranus and Neptune: presumably the former grew much faster and had much more gas in their extended atmospheres than the latter, which are essentially gas-poor, ice-giant planets. Thus, one would expect that both processes (i) and (ii) would have led to substantially different irregular satellite systems for these two pairs of planets.

The capture of irregular satellites in the framework of the Nice model has been investigated in details in Nesvorny et al. (2007). A characteristic feature of the Nice model is that, at the instability time, the giant planets suffered mutual close encounters. It was proposed in Nesvorny et al. (2007) that planetesimals wandering in the vicinity of the sites of such encounters could become trapped onto permanent orbits around the planets via gravitational three-body reactions. Numerical simulations showed that this process is effective, and leads to orbital distributions of satellites very similar to those observed around each planet. Moreover, assuming that the mass of the planetesimal disk was as in the Nice model and that the planetesimals had a size distribution 
similar to today's Kuiper belt, the capture efficiencies predict quite correctly the sizes of the largest irregular satellites around each planet. The current size distribution of irregular satellites, which is much shallower than that of the Kuiper belt, is then explained by their post-capture intense collisional evolution (Bottke et al., 2010).

From the results in Nesvorny et al. (2007), the capture of the irregular satellites of Saturn, Uranus and Neptune is a generic process because these planets experience planet-planet encounters in all the successful simulations of the Nice model. Instead, the capture of the satellites of Jupiter is not generic, because in most realization of the Nice model Jupiter does not encounter another planet. Only some of the successful simulations of the Nice model have Jupiter-Uranus or Jupiter-Neptune encounters. The fact that Jupiter has an irregular satellite system like that of the other planets argues that such encounters did happen in reality.

This conclusion is supported by the investigation of the orbital evolution of the terrestrial planets (Brasser et al., 2009) and of the asteroid belt (Morbidelli et al., 2010). These studies show that, in absence of encounters between Jupiter and another planet, the orbital separation between Jupiter and Saturn would have increased slowly and, consequently, the orbits of the terrestrial planets would have acquired too large eccentricities and the final orbital distribution in the asteroid belt would have become inconsistent with that observed. Instead, if Jupiter had had an encounter with an ice giant, the orbital separation between Jupiter and Saturn would have increased impulsively; this would have allowed the terrestrial planets to stay on moderate eccentricity orbits and the asteroid belt to avoid the formation of spurious empty regions within its boundaries.

\section{THE KUIPER BELT}


In the Nice model, the proto-planetary disk is assumed do have an outer edge at about $35 \mathrm{AU}$, otherwise Neptune ends its migration too far from its current orbital position. Proto-planetary disks often have sharp outer edges, as inferred from the radial distribution of dust in debris disks (the disk of AU Mic, for instance, is inferred to have an outer edge at $30 \mathrm{AU}$; Augereau andBeust, 2006). These edges might have been formed by several mechanisms, such as tidal truncation during early close stellar flybys (Kenyon an Bromley, 2004), photo-evaporation of the outer part of the proto-planetary disk (Adams et al., 2004), ineffective planetesimal accretion where the solid/gas ratio is too low (Youdin and Goodman, 2005). Thus, it is reasonable to assume that the planetesimal disk of the Solar System had an outer edge, but the assumption that this edge was at $35 \mathrm{AU}$ seems to be in conflict with the existence of a Kuiper belt between 35 and 50 AU. If the Nice model is correct, then there must be a mechanism to fill with objects an initially empty Kuiper belt.

Such mechanism was identified by Levison et al. (2008) with the temporary large eccentricity phase of Neptune at the time of the planetary instability. The point is that, when Neptune's orbit is eccentric, the full $(a, e)$ region up to the location of the $1 / 2$ resonance with the planet is chaotic. Thus, we can envision the following scenario. Assume, in agreement with several of the simulations of the Nice model, that the large eccentricity phase of Neptune is achieved when the planet has a semi-major axis of $\sim 28 \mathrm{AU}$, after its last encounter with Uranus. In this case, a large portion of the current Kuiper belt is already interior to the location of the $1 / 2$ resonance with Neptune. Thus, it is unstable, and can be invaded by objects coming from within the outer boundary of the disk (i.e. within $\sim 35 \mathrm{AU}$ ). When the eccentricity of Neptune damps out, the mechanism for the onset of chaos in the Kuiper belt region disappears. The Kuiper belt becomes stable, and the objects that happen to be there at that time remain trapped for the eternity. 
The simulations of Levison et al. (2008) successfully implanted a small fraction (approximately 1/1000) of the disk's planetesimals into the current Kuiper belt. This explains the low mass of the observed Kuiper belt population. The major success of the simulations is to reproduce the current sharp outer edge of the Kuiper belt, located at the position of the $1 / 2$ resonance with Neptune. This is the first, and so far only model, capable of explaining this characteristic of the belt. The observed orbital distribution in the Kuiper belt is also fairly well reproduced in the simulations, although the match is not perfect. For instance, there is a deficit in the synthetic population above 20 degrees of inclination.

\section{The new Nice model}

Despite of its successes, the original Nice model has some important weaknesses. The most important one is that the initial orbits of the giant planets are totally arbitrary. The assumption of small eccentricities and inclinations is reasonable, as this is expected from planet formation models, but the original orbital semi major axes are totally made up. In particular, Saturn and Jupiter are set initially on orbits close to their mutual $1 / 2$ resonance. The initial distance from this resonance is more or less tuned so to have an instability around the LHB time. Had this distance been larger, the planets would have not reached the resonance and would not have become unstable; had this distance been smaller, the resonance crossing would have occurred too early.

Clearly, there is the need to justify better the initial orbits of the planets. The initial conditions of the Nice model should correspond to the orbital structure that the Solar System had when it emerged from the gas-disk phase. Thus, the best way to set a valid initial orbital configuration of the planets is to study the dynamical evolution that said planets should have had when they were 
still embedded in the gas.

\subsection{The dynamics of the giant planets in a gas-disk}

The gravitational interaction of planets with a disk of gas leads to the orbital migration of the former, on a timescale and a radial range respectively much shorter and much wider than those characterizing the migration induced by the interaction with the planetesimals in a gas-less disk. The gas-driven migration is named "Type-I" for medium-mass planets like Uranus and Neptune that do not open a gap in the gas-disk around their orbits; it is named "Type-II" for giant planets like Jupiter and Saturn that do open at least partial gaps. Both migrations generically force the planetary orbits to shrink. The discovery of a large number of extra-solar giant planets on orbits with small radii (less than $1 \mathrm{AU}$; even less than $0.1 \mathrm{AU}$ in the case of the so-called "Hot Jupiters") is an empirical demonstration that radial migration occurs in real nature.

As stated in the introduction of this paper, whoever studies planet migration in gas-disks is confronted with crucial questions: why is Jupiter at 5 AU? Why did Jupiter not migrate closer to the Sun, unlike most of the known extra-solar planets?

The answer relies on the co-existence of Jupiter and Saturn, with their specific 3:1 mass ratio. In fact, as first showed in Masset and Snellgrove (2001) with hydro-dynamical simulations, Saturn migrates inwards faster than Jupiter and consequently it approaches the major planet until it is trapped in its $2 / 3$ mean motion resonance (where the orbital period of Jupiter is $2 / 3$ that of Saturn; see Fig 3). More recently, it has been shown (Pierens and Nelson, 2008) that the capture of a Saturn-like planet into the $2 / 3$ resonance with a Jupiter-like planet is a very robust outcome of simulations, independent of initial conditions and of the mass-growth history of the outer planet. 


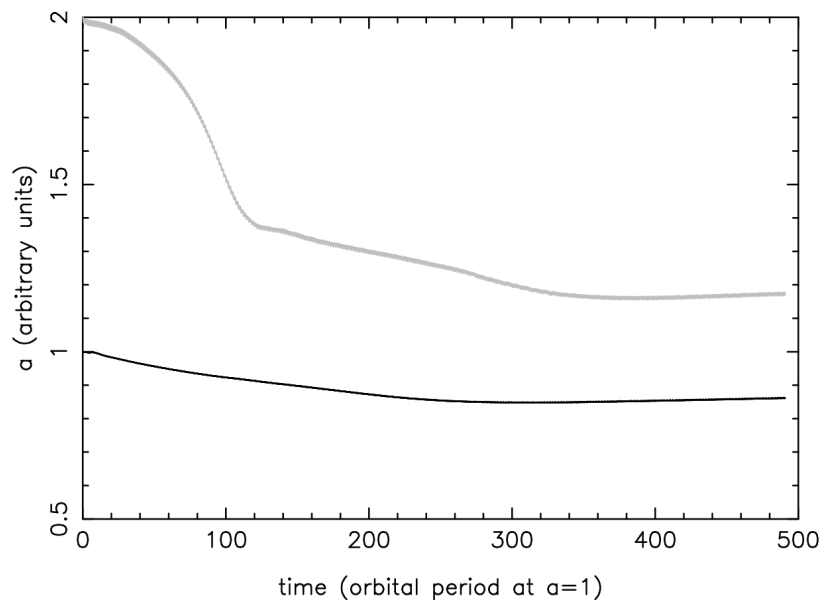

Fig. 3.- An illustration of the dynamical evolution of Jupiter and Saturn in the gas-disk, as in Masset and Snellgrove (2001). The black and grey curves show the evolutions of the semi major axes of Jupiter and Saturn, respectively. Capture in the 2/3 mean motion resonance occurs when the migration of Saturn is reversed.

Once in $2 / 3$ resonance configuration, the planets cease migrating inwards. It was shown in Morbidelli and Crida (2007) that the subsequent orbital evolution depends on the properties of the disk, particularly the scale height. In general, both planets migrate outwards together, on a short timescale. However, if the disk is very thick, the migration rate is very slow, as in Fig 3 . For some appropriate disk thickness there is essentially no migration. 2

The presence of asteroids inside of Jupiter's orbit suggests at first sight that Jupiter never

\footnotetext{
${ }^{2}$ Notice that, for two giant planets to avoid inward migration by this mechanism, it is essential that the mass of the outer planet is a fraction of the mass of the inner planet, as in the Jupiter-Saturn case (Masset and Snellgrove, 2001; Morbidelli and Crida, 2007). Planets of comparable masses or with a reversed mass ratio do migrate towards the central star also after resonance trapping.
} 
came closer to the Sun than its present position. Thus the parameters of the circum-solar disk should have been close to those resulting in a non-migrating evolution of the Jupiter-Saturn pair after their trapping in resonance. However, it has been proposed (Walsh et al., 2010) that Jupiter migrated down to 1.5 AU before Saturn formed and was captured in resonance; when this occurred, the two planets reversed migration and Jupiter reached 5.4 AU when the gas-disk disappeared. It has been argued that this kind of evolution would explain the properties of the terrestrial planets -in particular the large Earth/Mars mass ratio- and of the asteroid belt -in particular the dichotomy of physical properties of inner belt vs. outer belt asteroids- better than any other existing model. In either case, the $2 / 3$ resonance configuration of Saturn and Jupiter explains why Jupiter did not come to, or did not stay at, a small distance from the Sun.

The presence of the two major planets in a configuration characterized by no inward migration must have strongly influenced the dynamics of Uranus and Neptune and may even have played a role in their accretion. In fact, any sizeable proto-planet formed in the outer disk should have migrated inwards by Type I migration, until being trapped in some resonance with Saturn, or at the outer edge of its gap (Pierens and Nelson, 2008). The accumulation of embryos at specific sites outside the orbit of Saturn may have boosted the accretion of the cores of Uranus and Neptune. This phase, however, has never been modeled in details.

A search for possible orbital configurations of Uranus and Neptune relative to Jupiter and Saturn was done in Morbidelli et al. (2007), with a step-wise approach. First Jupiter and Saturn were set in a $2 / 3$ resonant, non-migrating orbital configuration. Then several hydro-dynamical simulations were done, placing Uranus at various orbital separations from Saturn and assuming a disk density close to the so-called "Minimum Mass Solar Nebula" (Weidenschilling, 1977). It was observed that Uranus migrated too fast to be trapped in the 1/2 resonance with Saturn. 
Conversely it could be trapped, depending on the initial conditions, in the $2 / 3$ or $3 / 4$ resonances. Configurations with Uranus closer to Saturn than the 3/4 resonance turned out to be unstable, with Uranus chased outwards by a distant encounter with Saturn, and eventually trapped in one of the two resonances listed above. Finally, for each of the two final stable configurations achieved by Uranus, a second set of hydro-dynamical simulations was done placing Neptune at various initial orbital separations from Uranus. It was observed that Neptune migrated too fast to be trapped in either the $1 / 2$ or $2 / 3$ resonances with Uranus. Instead, it could be trapped, depending on initial conditions, into the $3 / 4,4 / 5$ or $5 / 6$ resonances. Thus, in total 6 orbital configurations could be found, in which all planets are in resonance with each other. Other possible multi-resonant configurations of the giant planets have been found by Batygin and Brown (2010) using N-body integrations with forces that mimic Type-I migration of Uranus and Neptune.

\subsection{The dynamics of the giant planets after the gas-disk removal}

Many of the multi-resonant configurations described above are stable on Gy timescale once the gas-disk is removed. However, if there is a remnant planetesimal disk, the planet-planetesimals interactions perturb the orbits of the planets, and eventually may extract the planets from their mutual resonances. Resonances have a strong stabilizing effect for close orbits (a clear example is that of Pluto which, despite it crosses the orbit of Neptune, is stable because it is in its $2 / 3$ resonance). Once the planets are extracted from their mutual resonances, this stabilizing effect ends. The planets rapidly become unstable, because they are too close to each other. A phase of mutual scattering starts, similar to that described in the original Nice-model paper (Tsiganis et al., 2005; Gomes et al., 2005). The simulations in Morbidelli et al. (2007) and Batygin and 
Brown (2010) show that the final orbits that the planets achieve once the planetesimal disk is dispersed are often similar to the real ones. This shows that the multi-resonant configuration, which the giant planets should have been driven into during the gas-disk phase, can be consistent with the current orbital architecture of the planets, provided that the latter passed through a global instability phase.

Could this instability occur late, as in the original Nice model (Gomes et al., 2005), so to explain the origin of the LHB? A delayed instability can not be simply obtained by assuming that the planetesimal disk starts approximately 1 AU beyond the orbit of the furthermost planet, as in Gomes et al. (2005). In fact, the planets are now in resonances with each other, and the combination of resonance locking among the planets with the planet-planetesimal scattering process makes the instability time much more sensitive to the exact location of the disk's inner edge than in Gomes et al. (2005). Such an extreme sensitivity to the disk's parameters is, of course, problematic.

This problem, however, appears only in simulations which, like all those of the papers quoted above, assume that the planetesimals do not interact dynamically with each other. Instead, if self-interactions are taken into account, for instance assuming that there are a few 100s Pluto-mass objects in the disk perturbing each other and the other particles, then there is a net exchange of angular momentum between the planets and the disk, even if there are no close encounters between planets and planetesimals. In particular, the planets loose energy and momentum, i.e. they try to migrate towards the Sun (Levison et al., 2011). The orbits of the planets tend to approach each other. This is different from the case where planets scatter planetesimals, in which the planetary orbits tend to separate from each other. Remember, though, that the planets are in resonances; so the ratios between their semi major axes cannot change. In response, the planetary 
eccentricities slowly increase. This eventually drives some planets to pass through secondary or secular resonances, which destabilize the original multi-resonant configuration. Due to this process, the instability time is late in general: in the simulations of Levison et al. (2011) it occurs at a time ranging from $350 \mathrm{My}$ to over $1 \mathrm{~Gy}$ for disks with inner edge ranging from 15.5 to $20 \mathrm{AU}$ (Neptune is at $\sim 11.5 \mathrm{AU}$ in these simulations), with no apparent correlation between instability time and initial location of the inner edge of the disk.

Together, the papers by Morbidelli et al. (2007) and Levison et al. (2011) build the new version of the "Nice model". This is much superior than its original version (Tsiganis et al., 2005; Gomes et al., 2005) because (i) it removes the arbitrary character of the initial conditions of the planets by adopting as initial configuration one of the end-states of hydro-dynamical simulations and (ii) it removes the sensitive dependence of the instability time on the location of the inner edge of the disk; instead, a late instability seems to be a generic outcome.

\section{Conclusions}

According to our new understanding the evolution of the Solar System was characterized by three main "eras": In the gas-disk era, the giant planets acquired a multi-resonant configuration, in which each planet was in a mean-motion resonance with its neighbor. Given the Jupiter/Saturn mass-ratio, this prevented further inward migration, and explains why Jupiter was not closer than 5 AU from the Sun at the disappearance of the gas. It is possible that the giant planets had an inward-then-outward migration, bringing Jupiter temporarily at 1.5 AU (Walsh et al., 2010). At the disappearance of the gas, the system entered in the planetesimal-disk era. The orbits of the giant planets were at the time much closer to each other than they are now, and had significantly 
smaller eccentricities and inclinations. A massive disk of planetesimals persisted outside the orbit of the outermost giant planet. The gravitational interactions between the giant planets and this disk, slowly modified the resonant orbit of the former. Eventually, 600 My later, the giant planets became unstable, as a result of these slow orbital modifications. The chaotic phase that followed reshuffled the structure of the outer Solar System: the giant planets acquired their current orbits; most of the distant planetesimal disk was dispersed, causing the Late Heavy Bombardment of the terrestrial planets; a small fraction of the distant planetesimals got stranded in what we call today the Kuiper belt. With this profound re-organization, the Solar System entered into the current era, lasting since $~ 3.8$ Gy ago, in which it did not suffer any further significant change.

This is a radically different view with respect to the one that was consensual even just 10 years ago. However, it has a level of internal coherence and a consistency with the observed structure of the Solar System that have never been achieved before. This model describes a Solar System evolving under the same two main processes usually invoked to explain the structure of extra-solar planetary systems: radial migration in the gas-disk and global orbital instability. In fact, the simulations of the new Nice model, when they fail to reproduce our own system, often lead to planetary systems similar to some of those observed around other stars, with very eccentric planets or planets that remain in resonance forever. Thus, the great diversity among planetary systems seems to stem not from a diversity of processes, but from the diversity of outcomes under the same processes. This is due to the extreme sensitivity of the evolution to the initial and environmental conditions.

Nevertheless, the Nice story is not complete yet. It needs to be complemented with a model of the accretion of the giant planets that is consistent with their inferred dynamical evolution, which still does not exist. 


\section{References}

- F. C. Adams et al. Photoevaporation of Circumstellar Disks Due to External Far-Ultraviolet Radiation in Stellar Aggregates. The Astrophysical Journal 611 (2004) 360-379.

- J.C. Augereau, H. Beust. On the AU Microscopii debris disk. Density profiles, grain properties, and dust dynamics. Astronomy and Astrophysics 455 (2006) 987-999.

- K. Batygin, M.E. Brown, Early Dynamical Evolution of the Solar System: Pinning Down the Initial Condition of the Nice Model.The Astrophysical Journal 716 (2010) 1323-1331.

- W.F. Bottke et al. The Irregular Satellites: The Most Collisionally Evolved Populations in the Solar System. The Astronomical Journal 139 (2010) 994-1014.

- R. Brasser et al. Constructing the secular architecture of the Solar System II: the terrestrial planets. Astronomy and Astrophysics 507 (2009) 1053-1065.

- E.I. Chiang. Excitation of Orbital Eccentricities by Repeated Resonance Crossings: Requirements. The Astrophysical Journal 584 (2003) 465-471.

- E.I. Chiang, Y. Lithwick. Neptune Trojans as a Test Bed for Planet Formation. The Astrophysical Journal 628 (2005) 520-532.

- M. Ćuk, J.A. Burns. Gas-drag-assisted capture of Himalia's family. Icarus 167 (2004) 369-381.

- L. Dones et al. in M. Festou, H. A. Weaver, F. Keller (Eds.), Comets II, University Arizona Press., Tucson, 2004, p. 153-174. 
- M.J. Duncan, H.F. Levison. Scattered comet disk and the origin of Jupiter family comets. Science 276 (1997) 1670-1672.

- J.A. Fernandez, W.H. Ip. Some dynamical aspects of the accretion of Uranus and Neptune The exchange of orbital angular momentum with planetesimals. Icarus 58 (1984), 109-120.

- R.S. Gomes. Dynamical Effects of Planetary Migration on Primordial Trojan-Type Asteroids. The Astronomical Journal 116 (1998) 2590-2597.

- R.S. Gomes, A. Morbidelli, H.F. Levison. Planetary migration in a planetesimal disk: why did Neptune stop at 30 AU?. Icarus 170 (2004), 492-507.

- R. Gomes et al. Origin of the cataclysmic Late Heavy Bombardment period of the terrestrial planets. Nature 435 (2005) 466-469.

- J.M. Hahn, R. Malhotra. Orbital Evolution of Planets Embedded in a Planetesimal Disk. Astronomical Journal 117 (1999), 3041-3053.

- T.A. Heppenheimer, C. Porco. New contributions to the problem of capture. Icarus 30 (1977) 385-401.

- D.C. Jewitt, S.S. Sheppard. Physical Properties of Trans-Neptunian Object (20000) Varuna. Astronomical Journal 123 (2002) 2110-2120.

- S.J. Kenyon, B.C. Bromley. Stellar encounters as the origin of distant Solar System objects in highly eccentric orbits. Nature 432 (2004) 598-602.

- S.J. Kortenkamp. An efficient, low-velocity, resonant mechanism for capture of satellites by a protoplanet. Icarus 175 (2005) 409-418. 
- J.L. Lagrange. Essai sur le problme des trois corps. Oeuvres de Lagrange, Tome 6, Chapitre II. (1787) Gauthier-Villars. pp. 272-292.

- P.S. Laplace. Expositions du système du monde. (1796) Imprimerie Cercle-Social, Paris.

- H.F. Levison et al. Could the Lunar "Late Heavy Bombardment" Have Been Triggered by the Formation of Uranus and Neptune?. Icarus 151 (2001) 286-306.

- H.F. Levison et al. in Protostars and Planets V, University Arizona Press, Tucson, 2007, p. 669-684.

- H.F.Levison et al. Origin of the structure of the Kuiper belt during a dynamical instability in the orbits of Uranus and Neptune. Icarus 196 (2008) 258-273.

- H.F. Levison et al. Reevaluating the Early Dynamical Evolution of the Outer Planets. in preparation.

- F. Marzari et al. in W.F. Bottke et al. (Eds.) Asteroids III, University of Arizona Press., Tucson, 2002, p. 725-738.

- F. Masset, M. Snellgrove. Reversing type II migration: resonance trapping of a lighter giant protoplanet. Monthly Notices of the Royal Astronomical Society 320 (2001) 55-59.

- T.A. Michtchenko, C. Beaugé, F. Roig. Planetary Migration and the Effects of Mean Motion Resonances on Jupiter's Trojan Asteroids. Astronomical Journal 122 (2001) 3485-3491

- A. Morbidelli et al. Chaotic capture of Jupiter's Trojan asteroids in the early Solar System. Nature 435 (2005) 462-465. 
- A. Morbidelli, A. Crida. The dynamics of Jupiter and Saturn in the gaseous protoplanetary disk. Icarus 191 (2007) 158-171.

- A. Morbidelli et al. Dynamics of the Giant Planets of the Solar System in the Gaseous Protoplanetary Disk and Their Relationship to the Current Orbital Architecture. The Astronomical Journal 134 (2007) 1790-1798.

- A. Morbidelli et al. Constructing the secular architecture of the Solar System. I. The giant planets. Astronomy and Astrophysics 507 (2009) 1041-1052.

- A. Morbidelli et al. Evidence from the asteroid belt for a violent past evolution of Jupiter's orbit. Astron. J. (2010) in press.

- D. Nesvorný, D. Vokrouhlický, A. Morbidelli. Capture of Irregular Satellites during Planetary Encounters. The Astronomical Journal 133 (2007) 1962-1976.

- D.P. O’Brien, A. Morbidelli, W.F. Bottke. The primordial excitation and clearing of the asteroid belt - Revisited. Icarus 191 (2007) 434-452.

- A. Pierens, R.P. Nelson. Constraints on resonant-trapping for two planets embedded in a protoplanetary disc. Astronomy and Astrophysics 482 (2008) 333-340.

- E.W. Thommes, M.J. Duncan, H.F. Levison. The formation of Uranus and Neptune in the Jupiter-Saturn region of the Solar System. Nature 402 (1999) 635-638.

- K. Tsiganis et al. Origin of the orbital architecture of the giant planets of the Solar System. Nature 435 (2005) 459-461. 
- A.N. Youdin, J. Goodman. Streaming Instabilities in Protoplanetary Disks. The Astrophysical Journal 620 (2005) 459-469.

- K. Walsh et al. Origin of the Asteroid Belt and Mars' Small Mass. DPS (2010) 04.02

- G.W. Wetherill, G.R., Stewart. Formation of planetary embryos - Effects of fragmentation, low relative velocity, and independent variation of eccentricity and inclination. Icarus 106 (1993), 190-201.

- S.J. Weidenschilling. The distribution of mass in the planetary system and solar nebula. Astrophysics and Space Science 51 (1977) 153-158. 